\title{
Перераспределение атомов отдачи эрбия и кислорода и структура тонких приповерхностных слоев кремния, созданных высокодозной имплантацией аргона через поверхностные пленки $\mathrm{Er}$ и $\mathrm{SiO}_{2}$
}

\author{
(С) К.В. Феклистов ${ }^{1}$, А.Г. Черков ${ }^{1,2}$, В.П. Попов ${ }^{1}$, Л.И. Федина ${ }^{1}$ \\ ${ }^{1}$ Институт фризики полупроводников им. А.В. Ржанова \\ Сибирского отделения Российской академии наук, \\ 630090 Новосибирск, Россия \\ ${ }^{2}$ Новосибирский государственный университет, \\ 630090 Новосибирск, Россия \\ E-mail: kos@isp.nsc.ru
}

(Получена 4 апреля 2017 г. Принята к печати 2 апреля 2018 г.)

С использованием аналитической высокоразрешающей электронной микроскопии исследована структура $\mathrm{Si}$ и перераспределение атомов отдачи $\mathrm{Er}$ и О в тонких ( 10нм) приповерхностных слоях, внедренных с помощью имплантации $\mathrm{Ar}^{+}$с энергией 250-290 кэВ и дозой $1 \cdot 10^{16} \mathrm{~cm}^{-2}$ через пленки соответственно $\mathrm{Er}$ и $\mathrm{SiO}_{2}$ и последующего отжига. Установлено, что рекристаллизация $\mathrm{Si}$ срывается на расстоянии $\sim 20$ нм от поверхности, где достигается критическое для срыва значение концентрации эрбия $5 \cdot 10^{19} \mathrm{~cm}^{-3}$ при $T=950^{\circ} \mathrm{C}$. Это опровергает общепринятую модель переноса атомов $\mathrm{Er}$ фронтом рекристаллизации в $\mathrm{SiO}_{2}$ на поверхности. Вместо этого показано, что перераспределение атомов отдачи О к исходному оксиду во время отжига при неподвижных атомах $\mathrm{Er}$ обеспечивает формирование поверхностно неоднородных фаз эрбия таким образом, что обогащенная кислородом фаза $\mathrm{Er}-\mathrm{Si}-\mathrm{O}$ оказывается сосредоточенной в оксиде, а обедненная фаза $\mathrm{Er}-\mathrm{Si}$ остается в $\mathrm{Si}$. Это объясняет частичную потерю внедренного $\mathrm{Er}$ после снятия оксида вместе с $\mathrm{Er}-\mathrm{Si}-\mathrm{O}$ фазой. Показано, что нетипичное для рекристаллизации (100)-Si образование большой плотности микродвойников (локально до $10^{13} \mathrm{~cm}^{-2}$ ) связано с образованием пузырей и кластеров $\mathrm{Ar}$.

DOI: $10.21883 /$ FTP.2018.13.46872.8601

\section{1. Введение}

Одним из перспективных направлений кремниевой технологии в сфере информационных приложений является интеграция оптических систем передачи данных с электронными вычислительными системами „на одной плате“ или даже в одной микросхеме (система-на-кристалле), что при успешной реализации может привести к гибридным оптоэлектронным и оптическим вычислительным системам [1-3]. Однако на этом направлении существует целый ряд нерешенных задач. Ключевой из них является задача создания эффективного источника излучения в телекоммуникационном диапазоне пропускания оптоволоконных линий 1.5 мкм по технологии, совместимой с кремниевой. В качестве одного из вариантов решения задачи рассматривается легирование кремния атомами эрбия [4-6]. У иона эрбия не заполнена внутренняя $4 f$ оболочка и его внутриатомный оптический переход $\mathrm{Er}^{3+}:{ }^{4} I_{13 / 2} \rightarrow{ }^{4} I_{15 / 2}$ имеет длину волны 1.54 мкм, которая попадает в диапазон наименьших потерь оптоволоконных линий. Основным преимуществом системы $\mathrm{Si}: \mathrm{Er}$ является полная совместимость с кремниевой технологией и возможность прямой накачки возбуждения $\mathrm{Er}$ при пропускании электрического тока, т. е. создания светодиода (СД), работающего в режиме электролюминесценции (ЭЛ) Er.

Начиная с работ Ennen по фотолюминесценции (ФЛ) $\mathrm{Er}$ в кремнии [7], этот вопрос широко и активно исследовался [4-6]. Однако, несмотря на значительные достижения, проблема высокой эффективности люми- несценции кремниевых светодиодов при комнатной температуре не решена. Она составляет $1.5 \cdot 10^{-4}$ для системы $\mathrm{Si}: \mathrm{ErO}_{x}[8]$ и $\simeq 1 \%$ для структур $\mathrm{Si}, \mathrm{SiGe}[9-11]$. Одной из причин низкой эффективности светодиодов является нерешенная задача сильного легирования кремния атомами эрбия в оптически активном состоянии. Несмотря на достигнутые высокие значения полной концентрации $\mathrm{Er}$ в $\mathrm{Si}\left(\sim 1 \cdot 10^{20} \mathrm{~cm}^{-3}\right)$ и электрически активных комплексов $\mathrm{ErO}_{x} \sim 1 \cdot 10^{19}[12,13]$, оценочная концентрация оптически активных центров $\mathrm{Er}$ в $\mathrm{Si}$ не превышает 1-2\% от общей концентрации $\operatorname{Er}[14,15]$, тогда как для создания волноводных усилителей и лазеров на основе Er требуется, по оценкам, порядка $10^{18} \mathrm{~cm}^{-3}$ активных центров в кремниевых волноводах [16] и $10^{20} \mathrm{~cm}^{-3}$ в диэлектрических [17]. Оптически активным центром эрбия в кремнии принято считать кластер, состоящий из атома $\mathrm{Er}$, окруженного 6-8 атомами $\mathrm{O}$ $\left(\mathrm{ErO}_{n}\right.$ при $\left.n=6-8\right)[4,6]$. Для этого используется совместное легирование атомами $\mathrm{Er}$ и О. Однако полной оптической активации атомов $\mathrm{Er}$ таким способом пока не удается достичь. Помимо этого, современный научный интерес акцентирован на легированном Er пористом кремнии [18], структурах с нанокристаллическим кремнием [19], в диэлектрических матрицах [20-22] и структурах на основе прямозонных полупроводников, легированных $\operatorname{Er}[23,24]$. Тем не менее продолжаются поиски путей оптимизации соотношения $\mathrm{Er}$ и $\mathrm{O}$ в комплексе $\mathrm{Er}: \mathrm{O}_{x}$ в кремнии $[25,26]$. Кроме того, рассматривается усиление ФЛ $\mathrm{Er}$ в $\mathrm{Si}$ за счет плазмонных эффектов на наночастицах металлов [27]. Отмечается 
также возможность применения мелкого легирования $\mathrm{Er}$ с невысокими концентрациями для создания источника одиночных фотонов [28].

В настоящей работе детально исследована проблема приповерхностного внедрения атомов отдачи $\mathrm{Er}$ и О при высокоэнергетичной имплантации аргона. Задача мелкого внедрения примесей чаще всего возникает на пути миниатюризации электронных приборов. В случаe $\mathrm{Er}$, приповерхностная локализация может позволить исследовать другие способы его возбуждения, например, горячими носителями в канале МДП транзистора или при последовательной перезарядке связанного с Er уровня в МДП конденсаторной структуре. Попытка создания мелких слоев $\mathrm{Si}$ с концентрацией $\mathrm{Er}$, достигающей $5 \cdot 10^{20} \mathrm{~cm}^{-3}$, методом имплантации атомов отдачи при внедрении $\mathrm{Ar}^{+}$была продемонстрирована нами ранее в $[29,30]$. Однако в результате отжига образцов в среде $\mathrm{N}_{2}$ и последующего снятия оксида только половина внедренного $\mathrm{Er}$ оставалась в $\mathrm{Si}$, который при этом не проявлял оптической активности [29]. Предполагалось, что перенос $\mathrm{Er}$ в оксид во время отжига происходит по известному механизму сегрегации примеси на фронте рекристаллизации $\mathrm{Si}$ [31-33]. В [30] нами был предложен другой механизм, связанный с внутренним ростом толщины оксида за счет притока внедренных атомов отдачи О. Однако доказательств, однозначно свидетельствующих в пользу первого или второго механизма, на том этапе работ получено не было. В результате полученных в настоящей работе данных, основанных на использовании высокоразрешающей электронной микроскопии (ВРЭМ) и рентгеновской энергодисперсионной спектроскопии (EDS), позволяющей проведение химического анализа распределения примеси в слоях нанометрового масштаба, получены однозначные доказательства в пользу второго механизма.

\section{2. Методика эксперимента}

Для приготовления образцов были использованы пластины $\mathrm{Cz}-\mathrm{Si}(100)$ КДБ-10. В методе внедрения атомов отдачи $[29,30]$ последовательно, сначала через напыленную пленку $\mathrm{Er}$ толщиной 50 нм были имплантированы ионы $\mathrm{Ar}^{+}$с энергией 290 кэВ и дозой $1 \cdot 10^{16} \mathrm{~cm}^{-2}$, a затем через PECVD пленку $\mathrm{SiO}_{2}$ толщиной $50 \mathrm{Hм}$ имплантированы ионы $\mathrm{Ar}^{+} 250$ кэВ с дозой $1 \cdot 10^{16} \mathrm{~cm}^{-2}$. После этого, сохраняя осажденный $\mathrm{SiO}_{2}$ на поверхности, образцы отжигались при температуре $950^{\circ} \mathrm{C}$ в течение 1 ч в атмосфере $\mathrm{N}_{2}$.

Структурные исследования методом просвечивающей электронной микроскопии (ПЭМ), в том числе микроскопии высокого разрешения (ВРЭМ), выполнены на поперечных срезах образцов в сечении (110) с использованием аналитического микроскопа JEM 2200FS с корректором сферической аберрации. Микроскоп оборудован приставкой EX230BU для рентгеновской энергодисперсионной спектроскопии (energy dispersive $X$-ray spectroscopy (EDS)), позволяющей проводить химический анализ в режиме картирования (сканирования по поверхности образца). EDS-анализ распределения элементов был выполнен для $\operatorname{Er} L-, \mathrm{O}$ и $\mathrm{Si} K$-оболочек. Поверхностный слой $\mathrm{SiO}_{2}$ был удален в растворе $\mathrm{HF}$ перед большинством представленных измерений, если другое не оговорено специально в тексте.

\section{3. Экспериментальные результаты}

\section{1. ПЭМ характеризация структуры слоев, созданных имплантацией $\mathrm{Ar}^{+}$}

На рис. 1 представлены светлопольные дифракционные изображения в просвечивающем электронном микроскопе (ПЭМ изображения) в поперечном (110) сечении образца: сразу после имплантации ионов $\mathrm{Ar}^{+}$ через пленки $\mathrm{Er}$ и $\mathrm{SiO}_{2}$ (рис. 1, $a$ ) и последующего термического отжига (рис. $1, b, c)$. Для определения влияния имплантированных атомов аргона и атомов отдачи на структуру глубоких и приповерхностных слоев кремния на ПЭМ изображения наложены рассчитанный с помощью программы SRIM [34] профиль имплантированного $\operatorname{Ar}$ (рис. 1, a) и экспериментальные [29,30] профили распределения атомов отдачи эрбия до (рис. 1, $a$ ) и после (рис. $1, b)$ отжига. Как видно, при имплантации ионов $\mathrm{Ar}^{+}$формируется сплошной аморфный слой кремния $(a-\mathrm{Si})$, простирающийся от поверхности на глубину до 290 нм. Кроме того, видно, что вблизи поверхности формируется тонкий контрастный слой толщиной около 10 нм, который, как будет показано далее с помощью EDS-анализа, содержит атомы Er и O.

Высокотемпературный отжиг при $T=950^{\circ} \mathrm{C}$ приводит к рекристаллизации аморфного кремния, который сопровождается образованием различного типа и плотности протяженных дефектов (рис. $1, b, c)$, что определяется процессами, протекающими внутри сильно неоднородного имплантированного слоя. За границей аморфного слоя $(a-\mathrm{Si})$ формируются дислокационные петли Франка и полные петли, связанные с возникающим в этой области избытком межузельных атомов в $\mathrm{Si}$ (т.н. End of Range (EOR) дефекты) [35-37]. В то же время на глубине $\sim 200 \mathrm{Hм}$, чуть меньше среднего проецированного пробега ионов $\mathrm{Ar}^{+}\left(R_{p} \sim 220 \mathrm{Hм}\right.$ за вычетом толщины поверхностных пленок $50 \mathrm{Hм} \mathrm{Er}$ и $\mathrm{SiO}_{2}$ ), образуются микродвойники с высокой плотностью (рис. $1, b)$. Эти дефекты не связаны с кластеризацией собственных точечных дефектов, а обусловлены процессом твердофазной рекристаллизации в присутствии большой концентрации Ar (рис. 1,c) [35]. Кроме того, рекристаллизация становится зависящей и от концентрации атомов эрбия в приповерхностной области. На рис. $1, b$ на ПЭМ изображение наложен экспериментальный профиль распределения атомов $\mathrm{Er}$ после отжига $[29,30]$, который показывает, что его концентрация на глубине 20 нм, где останавливается рекристаллизация, 

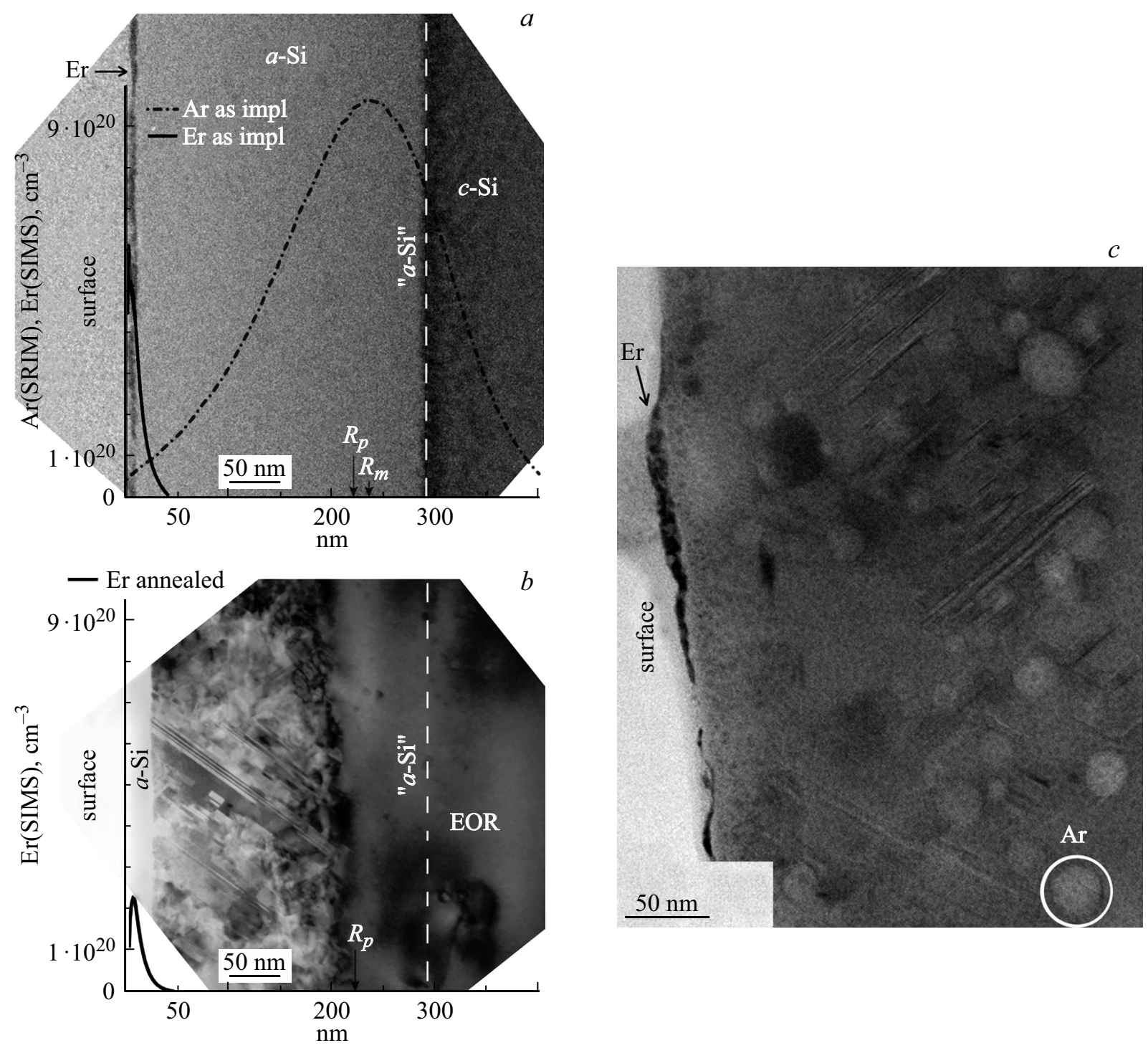

Рис. 1. ПЭМ изображения образцов в поперечном сечении (110) после имплантации ионов $\mathrm{Ar}^{+}(a)$ и последующего отжига $(b, c)$. На рисунках $(a)$ и $(b)$ показаны расчетный профиль распределения имплантированного аргона $(a)$ и экспериментальные профили атомов эрбия до $(a)$ и после $(b)$ отжига.

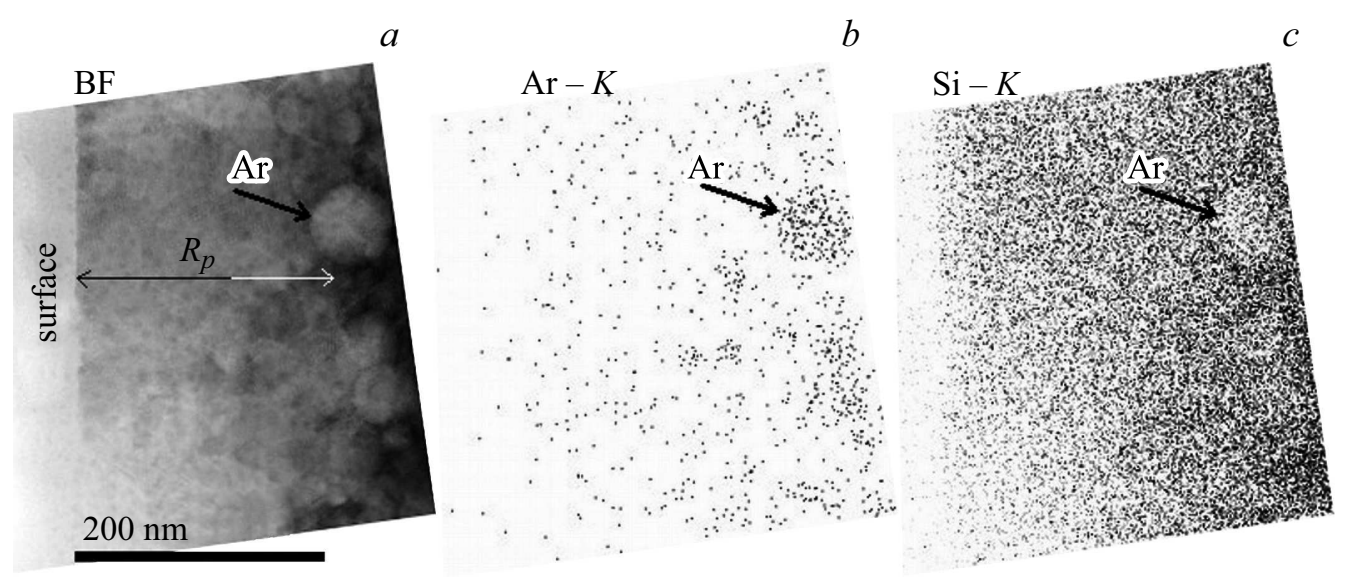

Pис. 2. a) [110]-ПЭМ изображение пузырей $\mathrm{Ar}$ в образце $\mathrm{Si}$ после имплантации ионов $\mathrm{Ar}^{+}$и последующей термообработки в сопоставлении с EDS картами распределения $\operatorname{Ar}(b)$ и $\mathrm{Si}(c)$ на данном участке. 
достигает $5 \cdot 10^{19} \mathrm{~cm}^{-3}$. Эта концентрация является критической для срыва процесса рекристаллизации $a$-Si при данной температуре отжига [31,32]. Из анализа рис. $1, b$ и $c$ также следует, что слой микродвойников начинается из области, где максимальная плотность пузырей. Она достигает порядка $10^{11} \mathrm{~cm}^{-2}$. Размеры пузырей варьируются от 10 до 30 нм в диаметре.

Из сопоставления ПЭМ изображения (рис. 2,a) с EDS картами распределения элементов $\mathrm{Ar}$ (рис. 2,b) и $\mathrm{Si}$ (рис. 2,c) следует, что круглые светлые пятна (дефицит материала) на рис. 2, $a$ совпадают с позициями избытка атомов $\mathrm{Ar}$ на рис. $2, b$ и с позициями дефицита атомов $\mathrm{Si}$ на рис. 2,c. Это наглядно подтверждает формирование пузырей, содержащих Ar. Один из крупных пузырей размером 40 нм в диаметре помечен стрелкой на рис. 2, $a$.

На рис. 3 представлены высокоразрешающие электронно-микроскопические (ВРЭМ) изображения микродвойников, простирающихся от области $R_{p}$ к поверхности. На вставке к рис. 3, $a$ представлена микродифракционная картина, полученная от области, содержащей двойники. Самые яркие рефлексы в углах ромбов соответствуют отражениям электронов в кристаллической решетке кремния с ориентацией (110). На каждом отрезке между этими рефлексами наблюдается по два более тусклых рефлекса, которые делят отрезок на три равные части и соответствуют рефлексам от микродвойников [38]. Тот факт, что микродвойники начинаются в слое с максимальной плотностью пузырей, соответствующей максимуму вводимых ими упругих деформаций, указывает на их роль в возникновении скользящих дислокаций Шокли, обеспечивающих формирование двойников при рекристаллизации аморфного слоя. Из рис. $3, a, b$ можно видеть, что локально дислокации Шокли скользят через каждые 4-10 плоскостей $\{111\}$, так что плотность микродвойников превышает $10^{13} \mathrm{~cm}^{-2}$.

Рис. $3, b$ также подтверждает, что слой микродвойников не доходит до поверхности на $\sim 20 \mathrm{Hм}$, где в условиях ВРЭМ отчетливо визуализируется аморфный кремний. Поверхность образца идентифицируется по наличию темного несплошного слоя эрбия (рис. $3, b$ ).

\subsection{EDS-анализ перераспределения атомов $\mathrm{Er}$ и О при отжиге}

Распределение химических элементов $\operatorname{Er}$ и О в тонком приповерхностном слое $\mathrm{Si}$, созданном атомами отдачи, исследовано с помощью EDS-анализа в поперечном сечении образцов (110). На рис. 4 представлены ПЭМ изображения в сопоставлении с EDS картами распределения $\mathrm{Er}$ и $\mathrm{O}$ на разных стадиях подготовки образцов: 1) сразу после внедрения атомов отдачи $\mathrm{Er}$ и $\mathrm{O}$ и удаления оксида с поверхности (рис. 4, $a$ ), 2) после отжига с сохранением оксида на поверхности (рис. 4, $b$ ), 3 ) после отжига и удаления слоя оксида (рис. $4, c$ ).

Видно, что до отжига в приповерхностной области формируется тонкий (10 нм) сплошной слой, содержащий высокую концентрацию атомов отдачи $\mathrm{Er}$ и $\mathrm{O}$ (рис. 4, $a-\mathrm{Er}-L, \mathrm{O}-K)$. EDS-анализ после отжига и сохранения оксида на поверхности показывает рост толщины оксида, так что слой эрбия становится почти полностью локализованным внутри оксида (рис. $4, b-$ $\mathrm{O}-K$ и $\mathrm{Er}-L)$. Это прямо указывает на перераспределение кислорода внутри слоя внедрения, которое, однако, не является простой диффузией кислорода. Из-за очень большой концентрации неподвижного Er отток кислорода к оксиду сопровождается образованием различающихся эрбиевых фаз: обогащенных $(\mathrm{Er}-\mathrm{Si}-\mathrm{O})$ и обедненных $(\mathrm{Er}-\mathrm{Si})$ по кислороду. Этот процесс носит локально и латерально сильно неоднородный характер, что отчетливо видно после удаления оксида (рис. 4,c). Теперь в приповерхностной области отсутствует не только сигнал по кислороду, но и частично по эрбию (рис. 4, $c$ $\mathrm{O}-K$ и $\mathrm{Er}-L)$, а рельеф поверхности становится очень развитым. Остаточная фаза $\mathrm{Er}-\mathrm{Si}$, как видно, формирует (рис. 4,c-BF) отдельные области темного контраста. Это объясняет результат, полученный методом МСВИ в $[29,30]$, что после отжига и снятия оксида лишь около половины атомов Er оставалось в приповерхностной области $\mathrm{Si}$, поскольку остальная часть $\mathrm{Er}$ удалялась вместе с оксидом в растворе НF перед МСВИ измерениями.

\section{4. Обсуждение}

\section{1. Формирование микродвойников}

Максимальная плотность микродвойников реализуется в области, где достигается и максимальная плотность пузырей Ar, и приводящий к двойникованию соответствующий им максимум упругих деформаций (рис. $1, b)$, необходимых для реализации скольжения дислокаций Шокли. Вопросы образования двойников в Si были детально исследованы теоретически на ранних этапах развития молекулярно-лучевой эпитаксии $[39,40]$, где было показано, что двойникование в напряженной гетеросистеме зависит от ориентации подложки и величины упругих напряжений. В условиях твердофазной кристаллизации формирование микродвойников также зависит от ориентации подложки $[35,38]$ и практически не наблюдается для $\mathrm{Si}(100)$ [35]. Для их появления на подложках с ориентацией (100) необходимы дефекты, связанные с сегрегацией примеси на фронте рекристаллизации [35]. Тот факт, что в нашем случае двойники возникают в слое с максимальной концентрацией пузырей, где достигаются максимальные деформации, мог бы свидетельствовать об их главенствующей роли в генерации скользящих дислокаций Шокли. Однако плотность пузырей составляет $\sim 10^{11} \mathrm{~cm}^{-2}$, тогда как плотность микродвойников локально достигает более чем $10^{13} \mathrm{~cm}^{-2}$, когда скольжение дислокаций Шокли осуществляется через 4-10 плоскостей $\{111\}$ (рис. 3,a). Это указывает на существование других возможных причин их образования. По данным рентгеновской фотоэлектронной спектроскопии (РФЭС), в имплантированном большими дозами $\mathrm{Ar}$ кремнии наблюдается 

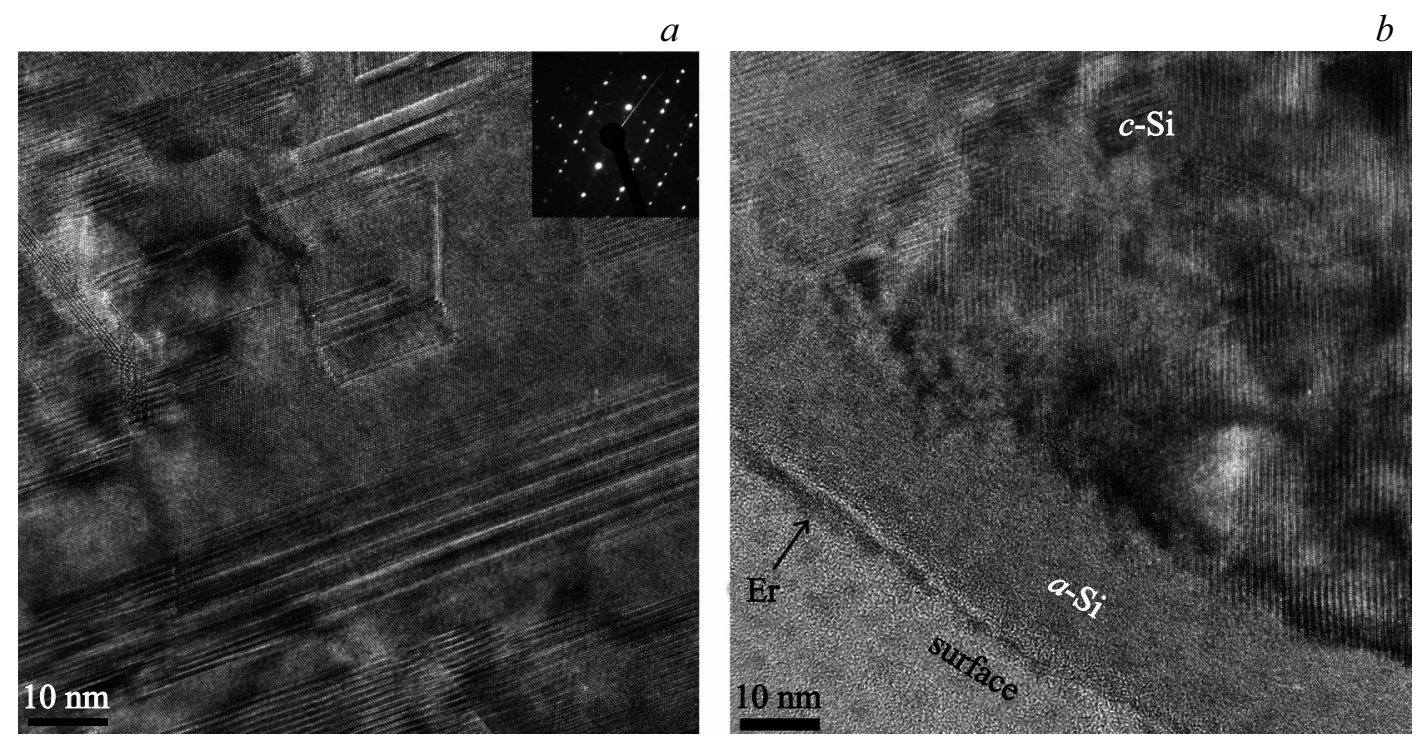

Рис. 3. а - ВРЭМ изображение микродвойниковых ламелей. На вставке дана микродифракционная картина. $b-$ ВРЭМ изображение аморфного слоя вблизи поверхности, где срывается рекристаллизация.

расщепление пика Ar2p, соответствующее только двум типам дефектов: одиночным атомам аргона и их агрегатам (кластерам) [41]. По всей видимости, именно кластеры Ar являются центрами, на которых происходит срыв бездефектной рекристаллизации с образованием микродвойников по модели [42]. При концентрации внедренного $\operatorname{Ar} 1 \cdot 10^{21} \mathrm{~cm}^{-3}$ (SRIM на рис. $\left.1, a\right)$ концентрация мелких кластеров $\mathrm{Ar}$ должна быть примерно на порядок меньше, что подтверждается методом атомно-зондовой томографии [43]. Объемная концентрация кластеров $\mathrm{Ar} 1 \cdot 10^{20} \mathrm{~cm}^{-3}$ в пересчете на плоскостную концентрацию составляет $2 \cdot 10^{13} \mathrm{~cm}^{-2}$, что по порядку величины соответствует наблюдаемой высокой плотности микродвойников превышающей $10^{13} \mathrm{~cm}^{-2}$, на рис. 3, $a$. Предполагается, что уже после формирования микродвойников кластеры $\mathrm{Ar}$ вырастают в размерах в течение отжига и становятся крупными пузырями (рис. $1, c, 2$ ), а их концентрация уменьшается в результате оствальдовского созревания. На сформировавшийся слой микродвойников этот процесс уже не влияет.

\section{2. Механизм перераспределения внедренных атомов отдачи Er и O при отжиге}

Полученные результаты по BРЭМ и EDS-анализу подтверждают данные МСВИ и фотолюминесценции [29] о том, что более половины внедренных атомов отдачи $\mathrm{Er}$ становятся частью оксида, существующего на поверхности при отжиге. Однако известный механизм переноса эрбия в оксид фронтом рекристаллизации, использованный в [29] для объяснения полученных результатов, оказался ошибочным. Детальный ВРЭМ анализ показывает, что рекристаллизация кремния далеко не доходит до поверхности и не может доставить эрбий к оксиду, существуюшему на поверхности (рис. $3, b$ ). Как видно из данного рисунка, вблизи поверхности остается слой аморфного кремния $(a-\mathrm{Si})$ толщиной 20 нм. Ранее Polman с соавт. показали $[31,32]$, что при достижении высокой концентрации $\mathrm{Er}$ в аморфном кремнии происходит срыв рекристаллизации. Согласно этим данным, срыв при $T=900^{\circ} \mathrm{C}$ происходит при концентрации $\mathrm{Er}$, равной $6 \cdot 10^{19} \mathrm{~cm}^{-3}$. В нашем случае при $T=950^{\circ} \mathrm{C}$ на глубине 20 нм, где реализуется срыв, концентрация $\mathrm{Er}$ составляет $5 \cdot 10^{19} \mathrm{~cm}^{-3}$, которая хорошо согласуется с [31,32].

Механизм диффузии $\mathrm{Er}$ из $a-\mathrm{Si}$ в оксид на поверхности также не работает, поскольку коэффициент его диффузии в $a-\mathrm{Si}$ очень низкий. В [29] коэффициент диффузии $\mathrm{Er}$ в кристаллическом кремнии $(c-\mathrm{Si})$ был оценен как менее чем $1 \cdot 10^{-15} \mathrm{~cm}^{2} / \mathrm{c}$ при $T=950^{\circ} \mathrm{C}$, а в $a$-Si он еще меньше (на несколько порядков) изза активного захвата $\operatorname{Er}$ на дефектах структуры [31,32]. Объяснение, предложенное без ключевых доказательств в [30], заключалось в перераспределении имплантированных атомов отдачи $\mathrm{O}$, которые очень подвижны при данной температуре отжига и в $c-\mathrm{Si}$, и в $a-\mathrm{Si}$ [44]. Проведенные ВРЭМ и EDS-анализ подтверждают, что перераспределение атомов О к существующему оксиду (рис. 4, $b$ ) обеспечивает увеличение его толщины за счет роста на гетерогранице. Поэтому неподвижный $\mathrm{Er}$ оказывается частично поглощен в оксиде, где он формирует обогащенную кислородом фазу $\mathrm{Er}-\mathrm{Si}-\mathrm{O}$. Возможно, что формируется смесь фаз $\mathrm{SiO}_{x}(x<2)$ и $\mathrm{Er}_{2} \mathrm{O}_{y} \quad(y \approx 3)$, поскольку энтальпия формирования у $\mathrm{Er}_{2} \mathrm{O}_{3}$ составляет -453.6 ккал/моль, тогда как у $\mathrm{SiO}_{2}$ -217.27 ккал/моль [45]. В подтверждение этому можно привести ряд работ, свидетельствующих о восстановлении эрбием оксида кремния и формировании $\mathrm{Er}_{2} \mathrm{O}_{3}$ или фазы $\mathrm{Er}-\mathrm{Si}-\mathrm{O}$ с высоким содержанием O [46-48]. 


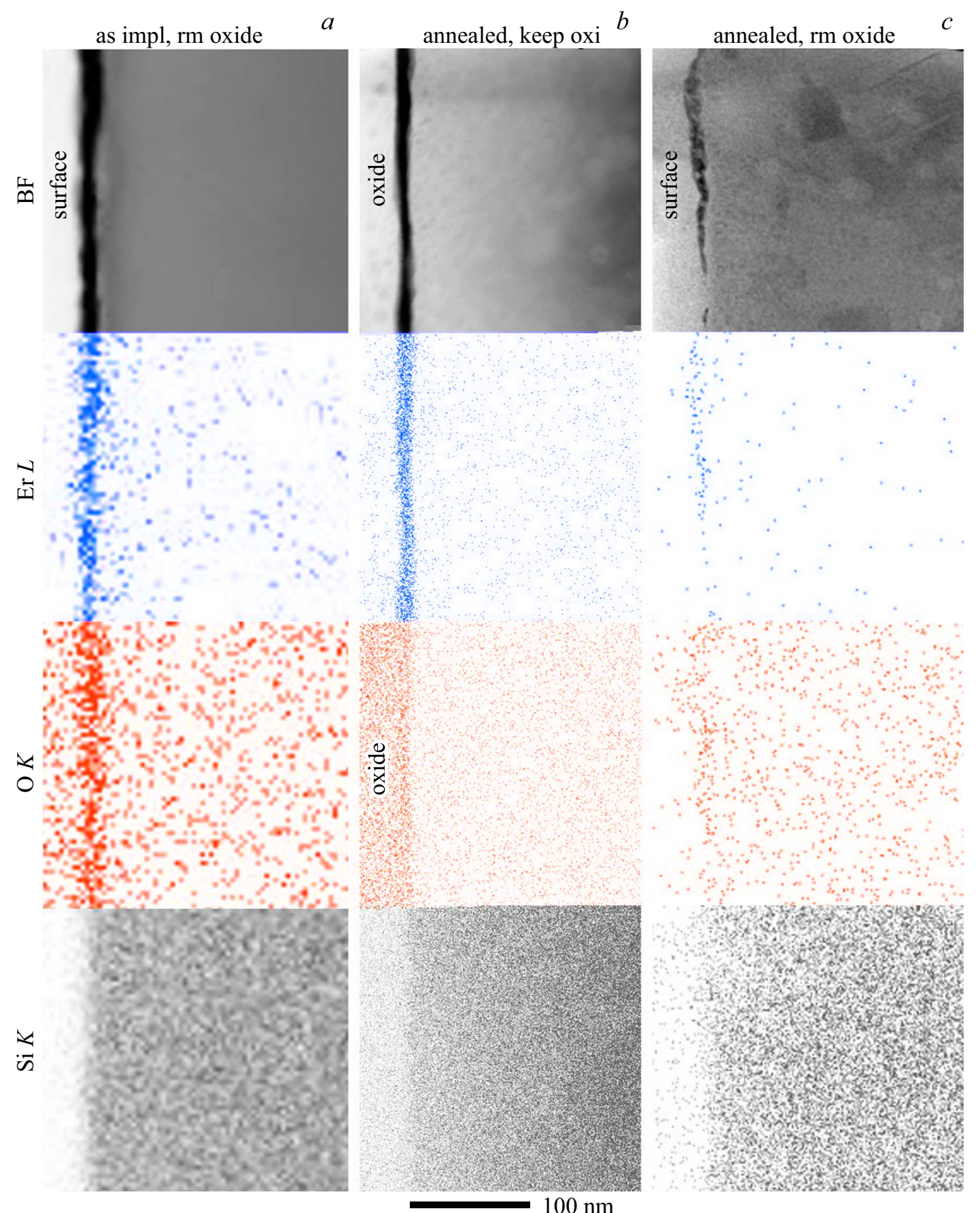

Рис. 4. ПЭМ изображения в сопоставлении с EDS картами распределения химических элементов $\mathrm{Er}$, O и $\mathrm{Si}$ в (110) сечении образцов на разных стадиях подготовки: $a-$ сразу после внедрения атомов отдачи $\mathrm{Er}$ и $\mathrm{O}, b-$ после отжига с сохранением $\mathrm{SiO}_{2}$, $c$ - после отжига и удаления $\mathrm{SiO}_{2}$ с поверхности. (Перепечатано с разрешения из [30], Copyright 2016 Elsevier Ltd.).

Однако, по всей видимости, внедренных атомов О оказалось недостаточно, чтобы полностью окислить весь содержащий $\mathrm{Er}$ слой $\mathrm{Si}$. Можно сделать следующую оценку. Если проинтегрировать расчетный профиль внедренного кислорода, представленный в [29,30], то получится интегральная плотность $5.3 \cdot 10^{15} \mathrm{O} / \mathrm{cm}^{2}$. Этого достаточно, чтобы сформировать слой 1 нм сплошного стехиометрического $\mathrm{SiO}_{2}$ с плотностью 2.65 г/см ${ }^{3}$ [49].
При латерально несплошном и нестехиометрическом $\mathrm{SiO}_{x}$ его толщина может быть пропорционально больше и достаточной, чтобы частично поглотить $\operatorname{Er}[29,30]$. To, что выросший за счет внедренного $\mathrm{O}$ слой $\mathrm{SiO}_{x}-$ латерально несплошной, подтверждается ПЭМ изображением поперечного среза (рис. 1,c), где видны развитый рельеф поверхности и остатки слоя, содержащего эрбий. В работах $[29,30]$ показано, что около 50\% 
внедренного $\mathrm{Er}$ теряется в приповерхностном окисле для одинаковой дозы внедренного О и разных доз внедренного Er. Это указывает на то, что около 50\% исходно гладкой границы $\mathrm{Si}-\mathrm{SiO}_{2}$ доокислилось и поглотило атомы Er. В результате после удаления оксида в приповерхностном слое $\mathrm{Si}$ остается фаза $\mathrm{Er}-\mathrm{Si}$ (рис. $1, c$ ), с низким содержанием О (рис. 4, $c-\mathrm{O}-K$ ). Таким образом, можно утверждать о формировании двух фаз в распадающемся твердом растворе из-за диффузии кислорода: фазы $\mathrm{Er}-\mathrm{Si}-\mathrm{O}$, которая становится частью поверхностного оксида, и фазы $\mathrm{Er}-\mathrm{Si}$, которая остается в кремнии. Предлагаемый механизм наилучшим образом объясняет потери внедренных атомов отдачи $\mathrm{Er}$ в оксиде при отжиге.

\section{5. Заключение}

При внедрении атомов отдачи $\mathrm{Er}$ и О в кремний с помощью последовательной имплантации $\mathrm{Ar}^{+}$через пленки $\mathrm{Er}$ и $\mathrm{SiO}_{2}$ соответственно удается достичь высокой концентрации $\operatorname{Er}\left(5 \cdot 10^{20} \mathrm{~cm}^{-3}\right)$ и О в приповерхностном слое кремния толщиной около 10 нм. Во время отжига происходит рекристаллизация аморфизованного имплантацией ионов $\mathrm{Ar}^{+}$слоя кремния, которая сопровождается образованием пузырей Ar и чрезвычайно большой плотности микродвойников, значительно превышающей плотность пузырей. Данный факт предполагает участие мелких кластеров $\mathrm{Ar}$ в инициации зарождения двойников. Рекристаллизация аморфизованного кремния срывается на глубине 20 нм от поверхности, где концентрация Er превышает критическое значение. Таким образом, весь $\mathrm{Er}$ в $\mathrm{Si}$ после отжига остается внутри аморфного слоя $\mathrm{Si}$, а не в кристаллической матрице.

Отжиг тонкого приповерхностного слоя Si с внедренными атомами отдачи $\operatorname{Er}$ и O при сохранении оксида на поверхности приводит к перераспределению кислорода к оксиду и образованию на гетерогранице двух фаз: обогащенной кислородом фазы $\mathrm{Er}-\mathrm{Si}-\mathrm{O}$, примкнувшей к оксиду, и обедненной кислородом фазы $\mathrm{Er}-\mathrm{Si}$ в кремнии. Из-за неоднородного латерального формирования фазы $\mathrm{Er}-\mathrm{Si}-\mathrm{O}$ вблизи оксида внутренняя граница раздела $\mathrm{Si}-\mathrm{SiO}_{x}$ становится сильно рельефной, так что при удалении оксида на поверхности кремния остаются отдельные области фазы $\mathrm{Er}-\mathrm{Si}$, содержащие около 50\% внедренного Er.

Авторы благодарят Исследовательский центр Новосибирского государственного университета за ПЭМ измерения в рамках государственной программы „Обеспечение проведения научных исследований“.

Работа выполнена при поддержке Программы фундаментальных исследований РАН (№ 8.1.5). Анализ дефектов структуры выполнен при поддержке проекта РНФ (№ 14-22-00143).

\section{Список литературы}

[1] H. Park, A.W. Fang, S. Kodama, J.E. Bowers. Opt. Express, 13 (23), 9460 (2005).

[2] A.W. Fang, H. Park, R. Jones, O. Cohen, M.J. Paniccia, J.E. Bowers. IEEE Phot. Techn. Lett., 18 (10), 1143 (2006).

[3] A.W. Fang, H. Park, O. Cohen, R. Jones, M.J. Paniccia, J.E. Bowers. Opt. Express, 14 (20), 9203 (2006).

[4] Н.А. Соболев. ФТП, 29 (7), 1153 (1995).

[5] A. Polman. J. Appl. Phys. 82, 1 (1997).

[6] A.J. Kenyon. Semicond. Sci. Technol., 20, R65 (2005).

[7] H. Ennen, J. Schneider, G. Pomrenke, A. Axmann. Appl. Phys. Lett., 43, 943 (1983).

[8] S. Coffa, G. Franzò, F. Priolo. J. Appl. Phys., 81, 2784 (1997).

[9] M.A. Green, J. Zhao, A. Wang, P.J. Reece, M. Gal. Nature, 412, 805 (2001).

[10] А.М. Емельянов, Н.А. Соболев. ФТП, 42, 336 (2008).

[11] N.A. Sobolev. Mater. Sci. Forum, 590, 79 (2008).

[12] S. Coffa, F. Priolo, G. Franzo, V. Bellani, A. Carnera, C. Spinella. Phys. Rev. B, 48, 11782 (1993).

[13] О.В. Александров, А.О. Захарьин, Н.А. Соболев, Ю.А. Николаев. ФТП, 36 (3), 379 (2002).

[14] A. Polman, G.N. van den Hoven, J.S. Custer, J.H. Shin, R. Serna, P.F.A. Alkemade. J. Appl. Phys., 77, 1256 (1995).

[15] O.B. Gusev, M.S. Bresler, P.E. Pak, I.N. Yassievich. Phys. Rev. B, 64, 075302 (2001).

[16] К.Е. Кудрявцев, Д.И. Крыжков, Л.В. Красильникова, Д.В. Шенгуров, В.Б. Шмагин, Б.А. Андреев, З.Ф. Красильник. Письма ЖЭТФ, 100, 913 (2014).

[17] J.D.B. Bradley, M. Pollnau. Laser \& Photon. Rev., 5 (3), 368 (2011).

[18] G. Mula, T. Printemps, C. Licitra, E. Sogne, F. D’Acapito, N. Gambacorti, N. Sestu, M. Saba, E. Pinna, D. Chiriu, P.C. Ricci, A. Casu, F. Quochi, A. Mura, G. Bongiovanni, A. Falqui. Scientific Rep., 7, 5957 (2017).

[19] J.M. Ramirez, Y. Berencen, L. Lopez-Conesa, J.M. Rebled, F. Peiro, B. Garrido. Appl. Phys. Lett., 103, 081102 (2013).

[20] S. Wang, A. Eckau, E. Neufeld, R. Carius, Ch. Buchal. Appl. Phys. Lett., 71, 2824 (1997).

[21] H. Krzyzanowska, K.S. Ni, Y. Fu, P.M. Fauchet. Mater. Sci. Eng. B, 177, 1547 (2012).

[22] Y. Berencen, S. Illera, L. Rebohle, J.M. Ramirez, R. Wutzler, A. Cirera, D. Hiller, J.A. Rodríguez, W. Skorupa, B. Garrido. J. Phys. D: Appl. Phys., 49, 085106 (2016).

[23] K. Dasari, J. Wu, H. Huhtinen, W.M. Jadwisienczak, R. Palai. J. Phys. D: Appl. Phys., 50, 175104 (2017).

[24] V.X. Ho, T.V. Dao, H.X. Jiang, J.Y. Lin, J.M. Zavada, S.A. McGill, N.Q. Vinh. Scientific Rep., 7, 39997 (2017).

[25] M.A. Lourenço, M.M. Miloševií, A. Gorin, R.M. Gwilliam, K.P. Homewood. Scientific Rep., 6, 37501 (2016).

[26] M.N. Drozdov, N.V. Latukhina, M.V. Stepikhova, V.A. Pokoeva, M.A. Surin. Modern Electronic Mater., 2, 7 (2016).

[27] S. Naczas, P. Akhter, M. Huang. Appl. Phys. Lett., 98, 113101 (2011).

[28] M. Celebrano, L. Ghirardini, P. Biagioni, M. Finazzi, Y. Shimizu, Y. Tu, K. Inoue, Y. Nagai, T. Shinada, Y. Chiba, A. Abdelghafar, M. Yano, T. Tanii, Enrico Prati. arXiv 1702.00331v1 (2017).

[29] К.В. Феклистов, Д.С. Абрамкин, В.И. Ободников, В.П. Попов. Письма ЖТФ, 41 (16), 52 (2015).

[30] K.V. Feklistov, A.G. Cherkov, V.P. Popov. Solid State Commun., 242, 41 (2016). 
[31] A. Polman, J.S. Custer, E. Snoeks, G.N. van den Hoven. Appl. Phys. Lett., 62, 507 (1993).

[32] J.S. Custer, A. Polman, H.M. van Pinxteren. J. Appl. Phys., 75, 2809 (1994).

[33] О.В. Александров, Ю.А. Николаев, Н.А. Соболев., ФТП, 32, 1420 (1998).

[34] J.F. Ziegler, J.P. Biersack, M.D. Ziegler. www.srim.org

[35] K.S. Jones, S. Prussin, E.R. Weber. Appl. Phys. A, 45, 1 (1988).

[36] B. de Mauduit, L. Laânab, C. Bergaud, M.M. Faye, A. Martinez, A. Claverie. Nucl. Instrum. Meth. B, 84, 190 (1994).

[37] F. Cristiano, J. Grisolia, B. Colombeau, M. Omri, B. de Mauduit, A. Claverie, L.F. Giles, N.E.B. Cowern. J. Appl. Phys., 87, 8420 (2000).

[38] M.D. Rechtin, P.P. Pronko, G. Foti, L. Csepregi, E.F. Kennedy, J.W. Mayer. Phil. Mag. A, 37, 605 (1978).

[39] A.L. Roitbijrd. Phys. Status Solidi A, 37, 329 (1976).

[40] A.K. Gutakovskii, S.I. Stenin, B.G. Zakharov. Phys. Status Solidi A, 67, 299 (1981).

[41] A.R. Lahrood, T. de los Arcos, M. Prenzel, A. von Keudell, J. Winter. Thin Sol. Films, 520, 1625 (2011).

[42] M. Prieto-Depedro, I. Romero, I. Martin-Bragado. Acta Materialia, 82, 115 (2015).

[43] M.K. Miller, R.G. Forbes. Atom-Probe Tomography: The Local Electrode Atom Probe (Springer, 2014).

[44] R.C. Newman. J. Phys.: Condens. Matter, 12, R335 (2000).

[45] National Bureau of Standards: Selected Values of Chemical Thermodynamic Properties (Pt. 7, Tech. Notes 270-7, p. 65; Pt. 2, p. 24).

[46] C. Choi, M. Jang, Y. Kim, M. Jun, T. Kim, M. Song. Appl. Phys. Lett., 91, 012903-1-3 (2007).

[47] C. Choi, M. Jang, Y. Kim, M. Jun, T. Kim, M. Song. Mater Trans., 51, 793 (2010).

[48] C.S. Wu, D.M. Scott, S.S. Lau. J. Appl. Phys., 58, 1330 (1985).

[49] Физические величины. Справочник под ред. И.С. Григорьева, Е.3. Мейлихова (М., Энергоатомиздат, 1991).

\section{Er and $O$ recoils redistribution and structure of thin surface Si layers made by means of high dose Ar implantation through $\mathrm{Er}$ and $\mathrm{SiO}_{2}$ surface films}

\author{
K.V. Feklistov ${ }^{1}$, A.G. Cherkov ${ }^{1,2}$, V.P. Popov ${ }^{1}$, \\ L.I. Fedina ${ }^{1}$
}

${ }^{1}$ Rzhanov Institute of Semiconductor Physics,

Siberian Branch of the Russian Academy of Sciences, 630090 Novosibirsk, Russia

${ }^{2}$ Novosibirsk State University, 630090 Novosibirsk, Russia

\begin{abstract}
The $\mathrm{Er}$ and $\mathrm{O}$ recoils redistribution and $\mathrm{Si}$ structure was investigated by means of analitical High Resolution Electron Microscopy (HREM) in thin $(10 \mathrm{~nm})$ surface Si layers made by means of $\mathrm{Ar}^{+} E=250-290 \mathrm{keV}, D=1 \cdot 10^{16} \mathrm{~cm}^{-2}$ implantation through $\mathrm{Er}$ and $\mathrm{SiO}_{2}$ surface films and annealing. It was found that $\mathrm{Si}$ recrystallisation stops at $20 \mathrm{~nm}$ from the surface, where $\mathrm{Er}$ concentration reaches critical value $5 \cdot 10^{19} \mathrm{~cm}^{-3}$ for $T=950^{\circ} \mathrm{C}$. This rejects the common mechanism of Er segregation into the cap oxide following the moving amorphous-crystalline interface during recrystallization. It was shown instead, that $\mathrm{O}$ recoils move toward cap oxide during the annealing and provide additional laterally nonuniform Si oxidation. Meanwhile Er recoil atoms stay immobile. This provides $\mathrm{O}$ rich phase $\mathrm{Er}-\mathrm{Si}-\mathrm{O}$ joints with cap oxide and $\mathrm{O}$ depleted phase $\mathrm{Er}-\mathrm{Si}$ stays in $\mathrm{Si}$. This explains the partial loss of implanted Er after removing of the cap oxide together with joint $\mathrm{Er}-\mathrm{Si}-\mathrm{O}$ phase. It was shown, that unusual for (100)-Si recrystallization formation of high density of microtwins (up to $10^{13} \mathrm{~cm}^{-2}$ locally) is associated with Ar clusters and bubbles formation.
\end{abstract}

